Homology, Homotopy and Applications, vol.19(1), 2017, pp.281-291

\title{
ČECH COMPLEXES FOR COVERS OF SMALL CATEGORIES
}

\author{
KOHEI TANAKA
}

(communicated by J.F. Jardine)

\begin{abstract}
We present a combinatorial analogue of the nerve theorem for covers of small categories, using the Grothendieck construction. We apply our result to prove the inclusion-exclusion principle for the Euler characteristic of a finite category.
\end{abstract}

\section{Introduction}

For an open cover $\mathcal{U}=\left\{U_{a}\right\}_{a \in A}$ of a space $X$, the Čech nerve $\check{C}(\mathcal{U})$ of $\mathcal{U}$ is a simplicial space defined by

$$
\check{C}(\mathcal{U})_{n}=\coprod_{a_{0}, \ldots, a_{n} \in A} U_{a_{0} \ldots a_{n}}
$$

where $U_{a_{0} \ldots a_{n}}$ denotes the intersection $U_{a_{0}} \cap \cdots \cap U_{a_{n}}$. Here, the face maps omit indices, and the degeneracy maps insert copies of indices. This is a simplicial space augmented over $X$, and its geometric realization is called the Cech complex of $\mathcal{U}$. Segal showed that the canonical map $|\check{C}(\mathcal{U})| \rightarrow X$ is a homotopy equivalence for a suitable cover $\mathcal{U}$ [Seg68]. On the other hand, Dugger and Isaksen proved that the natural map hocolim $\check{C}(\mathcal{U}) \rightarrow X$ from the homotopy colimit of the Cech nerve to the original space is a weak homotopy equivalence for any open cover [DI04]. In this paper, a combinatorial analogue of their result is provided for small categories. Let $C$ be a small category, and let $\mathcal{D}=\left\{D_{a}\right\}_{a \in A}$ be a collection of subcategories of $C$ satisfying $C=\bigcup_{a \in A} D_{a}$. We can define the $\check{C}$ ech nerve $\check{C}(\mathcal{D})$ of $\mathcal{D}$ as a simplicial object in the category of small categories, similarly to the above. In the context of small categories, the Grothendieck construction is known as a model of the homotopy colimit of a diagram in small categories [Tho79]. The Grothendieck construction $\operatorname{Gr}(\check{C}(\mathcal{D}))$ of the Čech nerve is also equipped with the natural functor $\operatorname{Gr}(\check{C}(\mathcal{D})) \rightarrow C$. A natural question to ask is when this induces a homotopy equivalence on classifying spaces.

Unfortunately, it does not hold for every cover. For example, let $C$ be a small category, formed as follows:

$$
x \longrightarrow y \longrightarrow z,
$$

with the terminal object $z$. If $D_{1}$ is the full subcategory with $\operatorname{ob}\left(D_{1}\right)=\{x, y\}$, and $D_{2}$ is the full subcategory with $\operatorname{ob}\left(D_{2}\right)=\{y, z\}$, then $C=D_{1} \cup D_{2}$. The classifying space

This work was supported by JSPS KAKENHI Grant Number 15K17535.

Received July 26, 2015, revised April 13, 2016; published on April 26, 2017.

2010 Mathematics Subject Classification: 55P10, 46M20.

Key words and phrases: nerve theorem, small category, inclusion-exclusion principle, Euler characteristic.

Article available at http://dx.doi.org/10.4310/HHA.2017.v19.n1.a14

Copyright (C) 2017, International Press. Permission to copy for private use granted. 
of $C$ is contractible; however, the classifying space of the Grothendieck construction for the Cech nerve of the cover $\left\{D_{1}, D_{2}\right\}$ is homotopy equivalent to a circle.

In order to solve the problem above, we introduce two classes of covers of small categories consisting of ideals and filters, respectively. These are generalized notions of ideals and filters of posets [Sta12, Zap98]. An ideal $D$ of a small category $C$ is a full subcategory such that an object $y$ of $C$ belongs to $D$, whenever $C(y, x) \neq \emptyset$ for some object $x$ of $D$. A cover $\mathcal{D}=\left\{D_{a}\right\}_{a \in A}$ of a small category $C$ is called an ideal cover, if $D_{a}$ is an ideal of $D$ for any $a \in A$. We can also define the dual notions; filters and filter covers.

Theorem 1.1. Let $\mathcal{D}$ be an ideal cover or a filter cover of a small category $C$. The natural functor $\operatorname{Gr}(\check{C}(\mathcal{D})) \rightarrow C$ induces a homotopy equivalence between their classifying spaces.

As an application of this result, we will show that we can obtain the inclusionexclusion principle of the Euler characteristics for finite categories with ideal covers or filter covers. The Euler characteristic $\chi(C)$ of a finite category $C$ is introduced by Leinster in [Lei08]. He calculates the Euler characteristic of the Grothendieck construction for a diagram in finite categories. By applying this to the (reduced) Čech nerve of a cover for a finite category, we obtain the following formula.

Theorem 1.2 (Inclusion-exclusion principle). Let $\mathcal{D}=\left\{D_{a}\right\}_{a \in A}$ be an ideal cover or a filter cover of a finite category $C$, indexed by a totally ordered finite set $A$. If each intersection $D_{a_{0} \ldots a_{i}}$ and $C$ have Euler characteristics, then we have

$$
\chi(C)=\sum_{i=0} \sum_{a_{0}<\ldots<a_{i}}(-1)^{i} \chi\left(D_{a_{0} \ldots a_{i}}\right) .
$$

Indeed, Leinster has demonstrated the inclusion-exclusion principle of the Euler characteristic for finite sets, as Example 3.4(d) in [Lei08]. The theorem above is a generalization of that result.

The rest of this paper is organized as follows. Section 2 describes the homotopy theory of Čech complexes, for covers of small categories. Subsequently, we focus on the inclusion-exclusion principle for Euler characteristics of finite categories in Section 3.

\section{The Čech nerve for covers of small categories}

Let $C$ be a small category, and let $\mathcal{D}=\left\{D_{a}\right\}_{a \in A}$ be a collection of subcategories of $C$ indexed by a set $A$. The intersection $\bigcap_{a \in A} D_{a}$ is a subcategory of $C$, whose set of objects is $\bigcap_{a \in A} \mathrm{ob}\left(D_{a}\right)$ and set of morphisms is $\bigcap_{a \in A} D_{a}(x, y)$, for each pair of objects $x$ and $y$.

On the other hand, the union $\bigcup_{a \in A} \mathcal{D}_{a}$ is a subcategory of $C$, whose set of objects is $\bigcup_{a \in A} \mathrm{ob}\left(D_{a}\right)$ and the set of morphisms is generated by compositions of morphisms of $D_{a}$. That is:

$$
\left(\bigcup_{a \in A} D_{a}\right)(x, y)=\left\{f_{n} \ldots f_{0} \mid f_{i} \in D_{a_{i}}\left(z_{i}, z_{i+1}\right), z_{0}=x, z_{n+1}=y\right\},
$$

for each pair of objects $x$ and $y$. 
Definition 2.1. Let $C$ be a small category. A collection of subcategories,

$$
\mathcal{D}=\left\{D_{a}\right\}_{a \in A}
$$

of $C$ is called a cover if $C=\bigcup_{a \in A} D_{a}$.

Definition 2.2 (Čech nerve). Let $\mathcal{D}=\left\{D_{a}\right\}_{a \in A}$ be a cover of a small category $C$. The $\check{C}$ ech nerve $\check{C}(\mathcal{D})$ is a simplicial object in the category Cat of small categories, defined as

$$
\check{C}(\mathcal{D})_{n}=\coprod_{a_{0}, \ldots, a_{n} \in A} D_{a_{0} \ldots a_{n}}
$$

where $D_{a_{0} \ldots a_{n}}$ denotes the intersection $D_{a_{0}} \cap \cdots \cap D_{a_{n}}$. Let us denote an object (a morphism) $x$ of $\check{C}(\mathcal{D})_{n}$ belonging to $D_{a_{0} \ldots a_{n}}$ by $x_{a_{0} \ldots a_{n}}$. The face map $d_{i}: \check{C}(\mathcal{D})_{n} \rightarrow$ $\check{C}(\mathcal{D})_{n-1}$ is the functor omitting the index $a_{i}$. That is:

$$
d_{i}\left(x_{a_{0} \ldots a_{n}}\right)=x_{a_{0} \ldots \hat{a_{i} \ldots a_{n}}},
$$

on objects and morphisms. Similarly, the degeneracy map $s_{j}: \check{C}(\mathcal{D})_{n} \rightarrow \check{C}(\mathcal{D})_{n+1}$ is the functor inserting the index $a_{j}$ between $a_{j}$ and $a_{j+1}$. That is:

$$
s_{j}\left(x_{a_{0} \ldots a_{n}}\right)=x_{a_{0} \ldots a_{j} a_{j} a_{j+1} \ldots a_{n}},
$$

on objects and morphisms.

In other words, $\check{C}(\mathcal{D})$ is a functor from $\Delta^{\text {op }}$ to the category Cat, where $\Delta$ consists of totally ordered finite sets $[n]=\{0, \ldots, n\}$ and order preserving maps. It takes $[n]$ to $\check{C}(\mathcal{D})_{n}$, and an order preserving map $\varphi:[m] \rightarrow[n]$ in $\Delta$ to the functor $\varphi^{*}: \check{C}(\mathcal{D})_{n} \rightarrow \check{C}(\mathcal{D})_{m}$. Here, $\varphi^{*}$ is given by $\varphi^{*}\left(x_{a_{0} \ldots a_{n}}\right)=x_{b_{0} \ldots b_{m}}$ on objects and morphisms, satisfying $b_{j}=a_{\varphi(j)}$ for each $j \in\{0, \ldots, m\}$.

A (pseudo-)functor from a small category to Cat provides a new small category, called the Grothendieck construction.

Definition 2.3 (Grothendieck construction). Let $C$ be a small category, and let $F: C \rightarrow$ Cat be a functor. The Grothendieck construction $\operatorname{Gr}(F)$ is a small category defined by the following:

- The set of objects consists of pairs $(c, x)$ of an object $c$ of $C$ and an object $x$ of $F(c)$.

- The set of morphisms $\operatorname{Gr}(F)((c, x),(d, y))$ consists of pairs of morphisms $(f, g)$, where $f \in C(c, d)$ and $g \in F(d)(F(f)(x), y)$.

- For each composable pair of morphisms

$$
\left(f_{1}, g_{1}\right) \in \operatorname{Gr}(F)((c, x),(d, y))
$$

and

$$
\left(f_{2}, g_{2}\right) \in \operatorname{Gr}(F)((d, y),(e, z))
$$

the composition is given by $\left(f_{2}, g_{2}\right)\left(f_{1}, g_{1}\right)=\left(f_{2} f_{1}, g_{2}\left(F\left(f_{2}\right)\left(g_{1}\right)\right)\right)$.

We can take the Grothendieck construction of the Cech nerve $\check{C}(\mathcal{D}): \Delta^{\text {op }} \rightarrow$ Cat for a cover $\mathcal{D}$ of a small category $C$. Let us see the details of this category. The set 
of objects of $\operatorname{Gr}(\check{C}(\mathcal{D}))$ consists of objects $x_{a_{0} \ldots a_{n}}$, indexed by $a_{0}, \ldots, a_{n} \in A$ and $n \geqslant 0$. The set of morphisms $\operatorname{Gr}(\check{C}(\mathcal{D}))\left(x_{a_{0} \ldots a_{n}}, y_{b_{0} \ldots b_{m}}\right)$ consists of pairs $\left(\varphi, f_{b_{0} \ldots b_{m}}\right)$ of an order preserving map $\varphi:[m] \rightarrow[n]$ such that $b_{j}=a_{\varphi(j)}$ for each $j$, and a morphism $f_{b_{0} \ldots b_{m}}: x_{b_{0} \ldots b_{m}} \rightarrow y_{b_{0} \ldots b_{m}}$ of $D_{b_{0} \ldots b_{m}}$. It is equipped with the canonical functor $\rho: \operatorname{Gr}(\check{C}(\mathcal{D})) \rightarrow C$ that eliminates indices. That is, $\rho\left(x_{a_{0} \ldots a_{n}}\right)=x$ on objects, and $\rho\left(\varphi, f_{a_{0} \ldots a_{n}}\right)=f$ on morphisms. A natural question is to ask when the functor $\rho$ induces a homotopy equivalence on classifying spaces. The classifying space $B C$ of a small category $C$ is constructed as the geometric realization of the nerve of $C$ (see [Seg68, Qui73, Hir03], for homotopical properties of classifying spaces). For a cover of a small category, the classifying space of the Grothendieck construction for the Čech nerve is called the Čech complex.

Definition 2.4. Let $C$ be a small category. An ideal $D$ of $C$ is a full subcategory such that an object $y$ of $C$ belongs to $D$, whenever $C(y, x) \neq \emptyset$ for some object $x$ of $D$. Dually, a filter $D$ of $C$ is a full subcategory such that an object $y$ of $C$ belongs to $D$, whenever $C(x, y) \neq \emptyset$ for some object $x$ of $D$. A cover $\mathcal{D}=\left\{D_{a}\right\}_{a \in A}$ of $C$ is called an ideal (a filter) cover if $D_{a}$ is an ideal (a filter) for any $a \in A$.

The notions above are generalizations of ideals and filters of posets [Sta12, Zap98]. Let $D$ be a full subcategory of a small category $C$. The category of complement $C \backslash D$ is defined as the full subcategory whose set of objects is $\operatorname{ob}(C) \backslash \mathrm{ob}(D)$. If $D$ is an ideal (a filter), then $C \backslash D$ is a filter (an ideal). In other words, the ideal $D$ yields a functor $C \rightarrow \mathcal{P}$, where $\mathcal{P}$ is the poset formed of $0<1$. The functor sends $\operatorname{ob}(D)$ to $\{0\}$ and $\mathrm{ob}(C \backslash D)$ to $\{1\}$. Conversely, for a functor $F: C \rightarrow \mathcal{P}$, the category of fiber $F^{-1}(0)$ is an ideal, and $F^{-1}(1)$ is a filter of $C$.

Theorem 2.5. Let $\mathcal{D}=\left\{D_{a}\right\}_{a \in A}$ be an ideal cover or a filter cover of a small category $C$. Then, the natural functor $\rho: \operatorname{Gr}(\check{C}(\mathcal{D})) \rightarrow C$ induces a homotopy equivalence between their classifying spaces.

Proof. We first consider the case in which $\mathcal{D}$ is an ideal cover. We use Quillen's theorem A [Qui73] for $\rho$. In order to apply this, we examine the left homotopy fiber $\rho \downarrow x$ of $\rho$, over an object $x$ of $C$. The set of objects consists of pairs $\left(y_{b_{0} \ldots b_{n}}, f\right)$ of an object $y_{b_{0} \ldots b_{n}}$ of $\operatorname{Gr}(\check{C}(\mathcal{D}))$, and a morphism $f: y \rightarrow x$ of $C$. The set of morphisms $(\rho \downarrow x)\left(\left(y_{b_{0} \ldots b_{n}}, f\right),\left(z_{c_{0} \ldots c_{m}}, g\right)\right)$ consists of morphisms $\left(\varphi, h_{c_{0} \ldots c_{m}}\right): y_{b_{0} \ldots b_{n}} \rightarrow z_{c_{0} \ldots c_{m}}$ of $\operatorname{Gr}(\breve{C}(\mathcal{D}))$, such that $g \circ h=f$.

On the other hand, we choose and fix an index $a \in A$ such that $x \in \operatorname{ob}\left(D_{a}\right)$. The set of objects of the over category $\operatorname{Gr}(\check{C}(\mathcal{D})) \downarrow x_{a}$ consists of morphisms $\left(v_{j}, f_{a}\right): y_{a_{0} \ldots a_{n}} \rightarrow$ $x_{a}$ of $\operatorname{Gr}(\check{C}(\mathcal{D}))$. Here, $v_{j}$ is the map $[0] \rightarrow[n]$ choosing $j \in\{0, \ldots, n\}$, and $f_{a}$ is a morphism from $y_{a_{j}}=y_{a}$ to $x_{a}$ of $D_{a}$.

The set of morphisms

$$
\left(\operatorname{Gr}(\check{C}(\mathcal{D})) \downarrow x_{a}\right)\left(\left(v_{j}, f_{a}\right),\left(v_{k}, g_{a}\right)\right)
$$

consists of morphisms $(\varphi, h)$ of $\operatorname{Gr}(\check{C}(\mathcal{D}))$, such that $\left(v_{k}, g_{a}\right) \circ(\varphi, h)=\left(v_{j}, f_{a}\right)$.

A functor

$$
F: \rho \downarrow x \longrightarrow \operatorname{Gr}(\check{C}(\mathcal{D})) \downarrow x_{a}
$$

is defined by $F\left(y_{a_{0} \ldots a_{n}}, f\right)=\left(v_{n+1}, f_{a}\right): y_{a_{0} \ldots a_{n} a} \rightarrow x_{a}$ on objects. Note that the 
object $y$ belongs to $D_{a}$, since $D_{a}$ is an ideal. A morphism

$$
\left(\varphi, h_{b_{0} \ldots b_{m}}\right):\left(y_{a_{0} \ldots a_{n}}, f\right) \rightarrow\left(z_{b_{0} \ldots b_{m}}, g\right)
$$

of $\rho \downarrow x$ is sent to a morphism $\left(\widetilde{\varphi}, h_{b_{0} \ldots b_{m} a}\right):\left(v_{n+1}, f_{a}\right) \rightarrow\left(v_{m+1}, g_{a}\right)$ of $\operatorname{Gr}(\check{C}(\mathcal{D})) \downarrow x_{a}$, where $\tilde{\varphi}:[m+1] \rightarrow[n+1]$ is the extension of $\varphi:[m] \rightarrow[n]$ with $\tilde{\varphi}(m+1)=n+1$. Conversely, a functor

$$
G: \operatorname{Gr}(\check{C}(\mathcal{D})) \downarrow x_{a} \longrightarrow \rho \downarrow x
$$

is defined by $G\left(v_{j}, g_{a}\right)=\left(y_{a_{0} \ldots a_{n}}, g\right)$, for an object $\left(v_{j}, g_{a}\right): y_{a_{0} \ldots a_{n}} \rightarrow x_{a}$ of the over category $\operatorname{Gr}(\check{C}(\mathcal{D})) \downarrow x_{a}$. There exists a natural transformation $t: G F \Rightarrow$ id defined by

$$
t\left(y_{a_{0} \ldots a_{n}}, f\right)=\left(d_{n+1}, \operatorname{id}_{a_{0} \ldots a_{n}}\right):\left(y_{a_{0} \ldots a_{n} a}, f\right) \longrightarrow\left(y_{a_{0} \ldots a_{n}}, f\right) .
$$

Note that the classifying space of the over category $\operatorname{Gr}(\check{C}(\mathcal{D})) \downarrow x_{a}$ is contractible, since it has a terminal object. We conclude that the classifying spaces of $\operatorname{Gr}(\check{C}(\mathcal{D})) \downarrow x_{a}$ and $\rho \downarrow x$ are homotopy equivalent and contractible. Quillen's theorem A completes the proof for the case of ideal covers.

If $\mathcal{D}$ is a filter cover of $C$, the opposite cover $\mathcal{D}^{\mathrm{op}}=\left\{D_{a}^{\mathrm{op}}\right\}_{a \in A}$ is an ideal cover of $C^{\mathrm{op}}$. Hence, the classifying space of the left homotopy fiber $\rho^{\prime} \downarrow x$ is contractible for the natural functor $\rho^{\prime}: \operatorname{Gr}\left(\check{C}\left(\mathcal{D}^{\text {op }}\right)\right) \rightarrow C^{\text {op }}$, and any object $x$ of $C^{\text {op }}$. The left homotopy fiber $\rho^{\prime} \downarrow x$ of $\rho^{\prime}$ coincides with the right homotopy fiber $x \downarrow \rho$ of $\rho$ for any object $x \in \mathrm{ob}(C)=\mathrm{ob}\left(C^{\mathrm{op}}\right)$. Again, Quillen's theorem A implies the result.

Thomason's homotopy colimit theorem [Tho79] tells us that the Cech complex for a cover $\mathcal{D}$ of a small category is homotopy equivalent to the homotopy colimit hocolim $B \check{C}(\mathcal{D})$ of the diagram $B \check{C}(\mathcal{D}): \Delta^{\text {op }} \rightarrow$ Top, in the category Top of spaces. The diagram $B \check{C}(\mathcal{D})$ is a Reedy cofibrant simplicial space [Hir03], with respect to the Strøm model structure on Top, since the degenerate part (the latching object) is a direct summand with open complement in each dimension. The Bousfield-Kan map hocolim $B \check{C}(\mathcal{D}) \rightarrow|B \check{C}(\mathcal{D})|$ is a homotopy equivalence by Theorem 18.7.4 of [Hir03]. Consequently, we have a homotopy equivalence

$$
|B \check{C}(\mathcal{D})| \simeq B C,
$$

for an ideal cover or a filter cover $\mathcal{D}$ of a small category $C$.

If the index set $A$ is equipped with a total order, then we can consider the ordered Čech nerve.

Definition 2.6 (Ordered Čech nerve). Let $\mathcal{D}=\left\{D_{a}\right\}_{a \in A}$ be a cover of a small category $C$, indexed by a totally ordered set $A$. The ordered $\check{C}$ ech nerve $\check{C}^{o}(\mathcal{D})$ is a simplicial object in Cat, defined as

$$
\check{C}^{o}(\mathcal{D})_{n}=\coprod_{a_{0} \leqslant \ldots \leqslant a_{n}} D_{a_{0} a_{1} \ldots a_{n}} .
$$

The face and degeneracy maps are defined similarly to the ordinary Čech nerve.

The ordered Čech nerve is smaller, and often easier to treat, than the ordinary Čech nerve. There exists an inclusion $\check{C}^{o}(\mathcal{D}) \rightarrow \check{C}(\mathcal{D})$ of simplicial objects in Cat. This induces a functor $I: \operatorname{Gr}\left(\check{C}^{o}(\mathcal{D})\right) \rightarrow \operatorname{Gr}(\check{C}(\mathcal{D}))$ between their Grothendieck constructions. 
Proposition 2.7. Let $\mathcal{D}=\left\{D_{a}\right\}_{a \in A}$ be a cover of a small category $C$, indexed by a totally ordered set $A$. The inclusion functor $I: \operatorname{Gr}\left(\check{C}^{o}(\mathcal{D})\right) \rightarrow \operatorname{Gr}(\check{C}(\mathcal{D}))$ induces a homotopy equivalence between their classifying spaces.

Proof. For an object $x_{a_{0} \ldots a_{n}}$ of $\operatorname{Gr}(\check{C}(\mathcal{D}))$, there exists a permutation $\sigma \in \Sigma_{n}$, such that $a_{\sigma(0)} \leqslant \ldots \leqslant a_{\sigma(n)}$ in $A$. In the case that $a_{i}=a_{j}$ for some $i<j$, we always choose $\sigma(i)<\sigma(j)$. This yields an inverse functor $J: \operatorname{Gr}(\check{C}(\mathcal{D})) \rightarrow \operatorname{Gr}\left(\check{C}^{o}(\mathcal{D})\right)$, sending $x_{a_{0} \ldots a_{n}}$ to $x_{a_{\sigma(0)} \ldots a_{\sigma(n)}}$ on objects. For a morphism $(\varphi, f): x_{a_{0} \ldots a_{n}} \rightarrow y_{b_{0} \ldots b_{m}}$ of $\operatorname{Gr}(\check{C}(\mathcal{D}))$, let $\sigma \in \Sigma_{n}$ and $\tau \in \Sigma_{m}$ be permutations for the canonical reordering of $a_{0} \ldots a_{n}$ and $b_{0} \ldots b_{m}$, respectively. For simplicity, denote $\alpha_{i}=a_{\sigma(i)}$ and $\beta_{j}=b_{\tau(j)}$, for each $i$ and $j$. For every $j \in\{0, \ldots, m\}$, we have

$$
\beta_{j}=b_{\tau(j)}=a_{\varphi \tau(j)}=\alpha_{\sigma^{-1} \varphi \tau(j)} .
$$

Consider the composition of maps $J(\varphi)=\sigma^{-1} \varphi \tau:[m] \rightarrow[n]$. We can describe

$$
b_{0} \ldots b_{m}=\overbrace{a_{0} \ldots a_{0}}^{\varphi^{-1}(0)} \overbrace{a_{1} \ldots a_{1}}^{\varphi^{-1}} \ldots \overbrace{a_{n} \ldots a_{n}}^{\varphi^{-1}(n)} .
$$

The permutation $\tau$ acts on $b_{0} \ldots b_{m}$ as $\sigma$ :

$$
b_{\tau(0)} \ldots b_{\tau(m)}=\overbrace{a_{\sigma(0)} \ldots a_{\sigma(0)}}^{\varphi^{-1}(\sigma(0))} \overbrace{a_{\sigma(1)} \ldots a_{\sigma(1)}}^{\varphi^{-1}(\sigma(1))} \ldots \overbrace{a_{\sigma(n)} \ldots a_{\sigma(n)}}^{\varphi^{-1}(\sigma(n))} .
$$

It follows that $J(\varphi)$ preserves order. The functor $J$ sends $(\varphi, f)$ to $(J(\varphi), f)$ on morphisms.

Trivially, we have $J I=\mathrm{id}$ on $\operatorname{Gr}\left(\check{C}^{o}(\mathcal{D})\right)$. Let us examine the composition $I J$. A functor $K: \operatorname{Gr}(\check{C}(\mathcal{D})) \rightarrow \operatorname{Gr}(\check{C}(\mathcal{D}))$ is defined by $K\left(x_{a_{0} \ldots a_{n}}\right)=x_{a_{0} \ldots a_{n} a_{\sigma(0)} \ldots a_{\sigma(n)}}$ on objects. For a morphism $(\varphi, f)$ of $\operatorname{Gr}\left(\check{C}^{o}(\mathcal{D})\right)$, define $K(\varphi, f)=(\varphi * J(\varphi), f)$, where $\varphi * J(\varphi):[2 m+1] \rightarrow[2 n+1]$ is given as

$$
\begin{cases}\varphi * J(\varphi)(i)=\varphi(i) & \text { if } 0 \leqslant i \leqslant m \\ \varphi * J(\varphi)(i)=J(\varphi)(i-m-1) & \text { if } m+1 \leqslant i \leqslant 2 m+1 .\end{cases}
$$

There exist natural transformations $t: K \Rightarrow I J$ with $t\left(x_{a_{0} \ldots a_{n}}\right)=\left(d_{0}^{n}, \mathrm{id}_{x}\right)$, and $s: K \Rightarrow$ id with $s\left(x_{a_{0} \ldots a_{n}}\right)=\left(d_{n+1} \ldots d_{2 n+1}, \operatorname{id}_{x}\right)$, for an object $x_{a_{0} \ldots a_{n}}$ of $\operatorname{Gr}(\check{C}(\mathcal{D}))$. Therefore, the induced maps from $I$ and $J$ on classifying spaces are homotopy inverses to each other.

For a cover $\mathcal{D}=\left\{D_{a}\right\}_{a \in A}$ of a small category $C$, indexed by a totally ordered set $A$, the natural functor $\rho^{o}: \operatorname{Gr}\left(\check{C}^{o}(\mathcal{D})\right) \rightarrow C$ is given as the composition $\rho^{o}=\rho I$. Theorem 2.5 and Proposition 2.7 imply the following.

Corollary 2.8. Let $\mathcal{D}=\left\{D_{a}\right\}_{a \in A}$ be an ideal cover or a filter of a small category $C$, indexed by a totally ordered set $A$. The natural functor $\rho^{o}: \operatorname{Gr}\left(\check{C}^{o}(\mathcal{D})\right) \rightarrow C$ induces a homotopy equivalence between their classifying spaces.

Note that the Grothendieck construction of the ordered Čech nerve is independent of the order on the index set. 
Proposition 2.9. Let $\mathcal{D}=\left\{D_{a}\right\}_{A}$ be a cover of a small category $C$, and let the index set $A$ be equipped with two total order $\leqslant_{1}$ and $\leqslant_{2}$. Denote the ordered Cech nerve induced from $\leqslant_{i}$ by $\check{C}^{o}(\mathcal{D})_{i}$ for $i=1,2$, respectively. Then, the Grothendieck constructions $\operatorname{Gr}\left(\check{C}^{o}(\mathcal{D})_{1}\right)$ and $\operatorname{Gr}\left(\check{C}^{o}(\mathcal{D})_{2}\right)$ are isomorphic to each other as categories.

Proof. Similarly to the proof of Proposition 2.7, we obtain a permutation $\sigma$ for an object $x_{a_{0} \ldots a_{n}}$ of $\operatorname{Gr}\left(\check{C}^{o}(\mathcal{D})_{1}\right)$, such that $x_{a_{\sigma(0)} \ldots a_{\sigma(n)}}$ belongs to $\operatorname{Gr}\left(\check{C}^{o}(\mathcal{D})_{2}\right)$. This yields a functor $F: \operatorname{Gr}\left(\check{C}^{o}(\mathcal{D})_{1}\right) \rightarrow \operatorname{Gr}\left(\check{C}^{o}(\mathcal{D})_{2}\right)$. We also obtain an inverse functor $G: \operatorname{Gr}\left(\check{C}^{o}(\mathcal{D})_{2}\right) \rightarrow \operatorname{Gr}\left(\check{C}^{o}(\mathcal{D})_{1}\right)$ by reordering the indexes. A straightforward calculation shows that $F G=$ id and $G F=$ id.

Next, we define the reduced Čech nerve, smaller than the ordered Čech nerve.

Definition 2.10 (Reduced Čech nerve). Let $\Delta_{i n j}$ denote the subcategory of $\Delta$, consisting of the same objects as $\Delta$ and injective order preserving maps. Let $\mathcal{D}=$ $\left\{D_{a}\right\}_{a \in A}$ be a cover of a small category $C$, indexed by a totally ordered set $A$. The reduced $\check{C}$ ech nerve of $\mathcal{D}$ is a functor $\check{C}^{o}(\widetilde{\mathcal{D}}): \Delta_{\text {inj }}^{\text {op }} \rightarrow$ Cat, defined by

$$
\check{C}^{o}(\widetilde{\mathcal{D}})_{n}=\coprod_{a_{0}<\ldots<a_{n}} D_{a_{0} a_{1} \ldots a_{n}} .
$$

This is the diagram in Cat where the degenerate parts of the ordered Cech nerve have been removed. It has only face maps omitting indices. Note that if $A$ is finite, then $\check{C}^{o}(\widetilde{\mathcal{D}})_{n}$ is the empty category for $n$ greater than the cardinality $A^{\sharp}$ of $A$.

The inclusion $\Delta_{i n j} \rightarrow \Delta$ induces a functor $L: \operatorname{Gr}\left(\check{C}^{o}(\widetilde{\mathcal{D}})\right) \rightarrow \operatorname{Gr}\left(\check{C}^{o}(\mathcal{D})\right)$ on their Grothendieck constructions.

Lemma 2.11. Let $\mathcal{D}=\left\{D_{a}\right\}_{a \in A}$ be a cover of a small category. For two morphisms $(\varphi, f),(\chi, g): x_{a_{0} \ldots a_{n}} \rightarrow y_{b_{0} \ldots b_{m}}$ of $\operatorname{Gr}(\check{C}(\mathcal{D}))$, if the indices $a_{0}, \ldots, a_{n}$ are distinguished, then $\varphi=\chi$.

Proof. For any $j \in\{0, \ldots, m\}$, the equality $a_{\varphi(j)}=b_{j}=a_{\chi(j)}$ holds. Since the indices $a_{0}, \ldots, a_{n}$ are distinguished, $\varphi(j)$ and $\chi(j)$ must be equal to each other.

The lemma above also holds for morphisms of $\operatorname{Gr}\left(\check{C}^{o}(\mathcal{D})\right)$ and $\operatorname{Gr}\left(\check{C}^{o}(\widetilde{\mathcal{D}})\right)$, since these are subcategories of $\operatorname{Gr}(\check{C}(\mathcal{D}))$.

Proposition 2.12. Let $\mathcal{D}=\left\{D_{a}\right\}_{a \in A}$ be a cover of a small category $C$, indexed by a totally ordered set $A$. The inclusion functor $L: \operatorname{Gr}\left(\check{C}^{o}(\widetilde{\mathcal{D}})\right) \rightarrow \operatorname{Gr}\left(\check{C}^{o}(\mathcal{D})\right)$ has a right adjoint functor.

Proof. For an arbitrary object $x_{a_{0} \ldots a_{n}}$ of $\operatorname{Gr}\left(\check{C}^{o}(\mathcal{D})\right)$, we obtain an object $x_{\alpha_{0} \ldots \alpha_{n^{\prime}}}$ of $\operatorname{Gr}\left(\check{C}^{o}(\widetilde{\mathcal{D}})\right)$ and a surjective order preserving map $\psi:[n] \rightarrow\left[n^{\prime}\right]$, such that $\alpha_{j}=a_{\psi(j)}$, by removing duplicate indices. This determines the reduction functor

$$
R: \operatorname{Gr}\left(\check{C}^{o}(\mathcal{D})\right) \rightarrow \operatorname{Gr}\left(\check{C}^{o}(\widetilde{\mathcal{D}})\right),
$$

sending $x_{a_{0} \ldots a_{n}}$ to $x_{\alpha_{0} \ldots \alpha_{n^{\prime}}}$ on objects. For a morphism $(\varphi, f): x_{a_{0} \ldots a_{n}} \rightarrow y_{b_{0} \ldots b_{m}}$ of $\operatorname{Gr}\left(\check{C}^{o}(\widetilde{\mathcal{D}})\right)$, the order preserving map $\varphi:[m] \rightarrow[n]$ induces $\varphi^{\prime}:\left[m^{\prime}\right] \rightarrow\left[n^{\prime}\right]$, making 
the following diagram commutative:

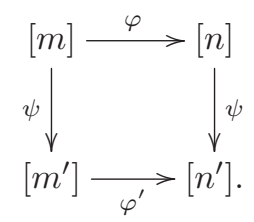

The reduction functor $R$ sends $(\varphi, f)$ to $\left(\varphi^{\prime}, f\right)$ on morphisms. We take objects $x_{a_{0} \ldots a_{n}}$ of $\operatorname{Gr}\left(\check{C}^{o}(\widetilde{\mathcal{D}})\right)$ and $y_{b_{0} \ldots b_{m}}$ of $\operatorname{Gr}\left(\check{C}^{o}(\mathcal{D})\right)$, respectively. Since $a_{0}, \ldots, a_{n}$ are distinguished, we have that

$$
\begin{aligned}
\operatorname{Gr}\left(\check{C}^{o}(\widetilde{\mathcal{D}})\right)\left(x_{a_{0} \ldots a_{n}}, R\left(y_{b_{0} \ldots b_{m}}\right)\right) & =\operatorname{Gr}\left(\check{C}^{o}(\widetilde{\mathcal{D}})\right)\left(x_{a_{0} \ldots a_{n}}, y_{\beta_{0} \ldots \beta_{m^{\prime}}}\right) \\
& \cong D_{\beta_{0} \ldots \beta_{m^{\prime}}}(x, y) \\
& =D_{b_{0} \ldots b_{m}}(x, y) \\
& \cong \operatorname{Gr}\left(\check{C}^{o}(\mathcal{D})\right)\left(L\left(x_{a_{0} \ldots a_{n}}\right), y_{b_{0} \ldots b_{m}}\right),
\end{aligned}
$$

by Lemma 2.11. Therefore, we conclude that the reduction functor $R$ is right adjoint to $L$.

The reduced Čech nerve is also equipped with the natural functor

$$
\tilde{\rho}=\rho^{o} L: \check{C}^{o}(\widetilde{\mathcal{D}}) \rightarrow C,
$$

the same as the ordered Čech nerve and the ordinary Čech nerve. Form Proposition 2.12 and Corollary 2.8, we can deduce the following.

Corollary 2.13. Let $\mathcal{D}=\left\{D_{a}\right\}_{a \in A}$ be an ideal cover or a filter cover of a small category $C$, indexed by a totally ordered set $A$. The natural functor $\tilde{\rho}: \check{C}^{o}(\widetilde{\mathcal{D}}) \rightarrow C$ induces a homotopy equivalence between their classifying spaces.

Definition 2.14. A cover $\mathcal{D}=\left\{D_{a}\right\}_{a \in A}$ of a small category $C$ is called locally finite when

$$
\left\{a \in A \mid x \in \mathrm{ob}\left(D_{a}\right)\right\}^{\sharp}<\infty,
$$

for any object $x$ of $C$.

Proposition 2.15. Let $\mathcal{D}=\left\{D_{a}\right\}_{a \in A}$ be a locally finite ideal cover of a small category $C$, indexed by a totally ordered set $A$. The natural functor $\tilde{\rho}: \operatorname{Gr}\left(\check{C}^{o}(\widetilde{\mathcal{D}})\right) \rightarrow C$ has a left adjoint functor.

Proof. A functor $\pi: C \rightarrow \operatorname{Gr}\left(\check{C}^{o}(\widetilde{\mathcal{D}})\right)$ is defined by $\pi(x)=x_{a_{0} \ldots a_{n}}$ on objects, where $D_{a_{0}}, \ldots, D_{a_{n}}$ are whole distinguished ideals in $\mathcal{D}$ that contain $x$. For a morphism $f: x \rightarrow y$ of $C$, the object $x$ belongs to an ideal $D$ if $y$ does. When we describe $\pi(x)=x_{a_{0} \ldots a_{n}}$ and $\pi(y)=y_{b_{0} \ldots b_{m}}$, the inclusion relation $\left\{b_{0}, \ldots b_{m}\right\} \subset\left\{a_{0}, \ldots, a_{n}\right\}$ holds. This yields an injection $\varphi:[m] \rightarrow[n]$, such that $b_{j}=a_{\varphi(j)}$. We define $\pi(f)=$ $(\varphi, f): \pi(x) \rightarrow \pi(y)$ on morphisms.

Take objects $x$ of $C$ and $y_{b_{0} \ldots b_{m}}$ of $\operatorname{Gr}\left(\check{C}^{o}(\widetilde{\mathcal{D}})\right)$, respectively. Then, by Lemma 2.11,

$$
C\left(x, \tilde{\rho}\left(y_{b_{0} \ldots b_{m}}\right)\right)=C(x, y)=D_{b_{0} \ldots b_{m}}(x, y) \cong \operatorname{Gr}\left(\check{C}^{o}(\widetilde{\mathcal{D}})\right)\left(\pi(x), y_{b_{0} \ldots b_{m}}\right) .
$$

Therefore, we conclude that the functor $\pi$ is left adjoint to $\tilde{\rho}$. 
When $\mathcal{D}=\left\{D_{a}\right\}_{a \in A}$ is a filter cover of a small category $C$, the opposite cover $\mathcal{D}^{\text {op }}=$ $\left\{D_{a}^{\mathrm{op}}\right\}_{a \in A}$ is an ideal cover of $C^{\text {op }}$. The following corollary then follows immediately.

Corollary 2.16. Let $\mathcal{D}=\left\{D_{a}\right\}_{a \in A}$ be a locally finite filter cover of a small category $C$, indexed by a totally ordered set $A$. The natural functor $\operatorname{Gr}\left(\check{C}^{o}\left(\widetilde{\mathcal{D}}^{\text {op }}\right)\right) \rightarrow C^{\text {op }}$ has a left adjoint functor.

\section{The inclusion-exclusion principle for the Euler character- istics of finite categories}

In this section, we focus on the inclusion-exclusion principle for the Euler characteristic of a finite category. The Euler characteristic of a finite category was introduced by Leinster [Lei08]. This is a generalization of Möbious inversion for posets [Rot64]. Let us briefly review the definition. In this paper, we use the rational numbers $\mathbb{Q}$ as the value of Euler characteristics of finite categories.

Definition 3.1. Suppose that $C$ is a finite category that has finitely many objects and morphisms.

1. The similarity matrix of $C$ is the function $\zeta: \mathrm{ob}(C) \times \mathrm{ob}(C) \rightarrow \mathbb{Q}$, given by the cardinality of the set of morphisms $\zeta(a, b)=C(a, b)^{\sharp}$.

2. Let $u: \operatorname{ob}(C) \rightarrow \mathbb{Q}$ denote the column vector with $u(a)=1$, for any object $a$ of $C$. A weighting on $C$ is a column vector $w: \operatorname{ob}(C) \rightarrow \mathbb{Q}$ such that $\zeta w=u$. Dually, a coweighting on $C$ is a row vector $v: \operatorname{ob}(C) \rightarrow \mathbb{Q}$, such that $v \zeta=u^{*}$, where $u^{*}$ is the transposed matrix of $u$.

Note that we have

$$
\sum_{i \in \mathrm{ob}(C)} w(i)=u^{*} w=v \zeta w=v u=\sum_{j \in \mathrm{ob}(C)} v(j),
$$

if both a weighting and a coweighting exist. Moreover,

$$
\sum_{i \in \mathrm{ob}(C)} w(i)=u^{*} w=v \zeta w=v \zeta w^{\prime}=u^{*} w^{\prime}=\sum_{i \in \mathrm{ob}(C)} w^{\prime}(i),
$$

for two (co)weightings $w$ and $w^{\prime}$ on $C$. This guarantees the following definition.

Definition 3.2. Let $C$ be a finite category. We say that $C$ has Euler characteristic if it has both a weighting $w$, and a coweighting $v$, on $C$. Then, the Euler characteristic of $C$ is defined by

$$
\chi(C)=\sum_{i \in \operatorname{ob}(C)} w(i)=\sum_{j \in \mathrm{ob}(C)} v(j) .
$$

It is well-known that the topological Euler characteristic $\chi_{T}$ has the following inclusion-exclusion formula:

$$
\chi_{T}(A \cup B)=\chi_{T}(A)+\chi_{T}(B)-\chi_{T}(A \cap B),
$$

for subcomplexes $A$ and $B$ of a finite $C W$-complex $X$. Recall the example given in 
our introduction. The finite category $C$ is

$$
x \longrightarrow y \longrightarrow z,
$$

with the terminal object $z, D_{1}$ is the full subcategory with $\operatorname{ob}\left(D_{1}\right)=\{x, y\}$, and $D_{2}$ is the full subcategory with $\operatorname{ob}\left(D_{2}\right)=\{y, z\}$. The Euler characteristic $\chi(C)=$ $\chi\left(D_{1} \cup D_{2}\right)=1$, since $C$ has a terminal object. However,

$$
\chi\left(D_{1}\right)+\chi\left(D_{2}\right)-\chi\left(D_{1} \cap D_{2}\right)=0+1-1=0 .
$$

The inclusion-exclusion principle does not hold in this case. In [FLS11], Fiore, Luck, and Sauer used the homotopy colimit of diagram in Cat, instead of the genuine colimit or union.

Now, we consider the following situation. Let $C$ be a finite category, and let $\mathcal{D}=$ $\left\{D_{a}\right\}_{a \in A}$ be a cover of $C$, indexed by a totally ordered finite set $A$. We denote the full subcategory of $\Delta_{i n j}$, consisting of $[n]$ for $0 \leqslant n \leqslant A^{\sharp}-1$ by $\Delta_{A^{\sharp}}$. The reduced Cech nerve $\check{C}^{o}(\widetilde{\mathcal{D}})$ can be regarded as a functor $\Delta_{A^{\sharp}}^{\mathrm{op}} \rightarrow$ Cat. The category $\Delta_{A^{\sharp}}^{\mathrm{op}}$ is a finite acyclic category, which never has circuit of morphisms. Moreover, each $\check{C}^{o}(\widetilde{\mathcal{D}})_{n}$ is a finite coproduct of finite categories. Leinster provided the product formula for the Euler characteristic of finite categories, with respect to Grothendieck construction in [Lei08]. By applying this to $\operatorname{Gr}\left(\check{C}^{\circ}(\widetilde{\mathcal{D}})\right)$, we obtain the inclusion-exclusion principle for ideal (filter) covers of a finite category.

Theorem 3.3 (Inclusion-exclusion principle). Let $\mathcal{D}=\left\{D_{a}\right\}_{a \in A}$ be an ideal cover or a filter cover of a finite category $C$, indexed by a finite set $A$. If each $D_{a_{0} \ldots a_{i}}$ and $C$ have Euler characteristics, then we have

$$
\chi(C)=\sum_{i=0}^{A^{\sharp}-1} \sum_{a_{0}<\ldots<a_{i}}(-1)^{i} \chi\left(D_{a_{0} \ldots a_{i}}\right) .
$$

Proof. Let $\mathcal{D}$ be an ideal cover of $C$. The category $\Delta_{A^{\sharp}}^{\mathrm{op}}$ has a unique weighting $w[n]=(-1)^{n}$ (see Example $3.4(\mathrm{~d})$ of [Lei08]). By applying Proposition 2.8 of [Lei08] to $\operatorname{Gr}\left(\check{C}^{o}(\widetilde{\mathcal{D}})\right)$, we have

$$
\chi\left(\operatorname{Gr}\left(\check{C}^{o}(\widetilde{\mathcal{D}})\right)\right)=\sum_{i=0}^{A^{\sharp}-1}(-1)^{i} \chi\left(\check{C}^{o}(\widetilde{\mathcal{D}})_{i}\right)=\sum_{i=0}^{A^{\sharp}-1} \sum_{a_{0}<\ldots<a_{i}}(-1)^{i} \chi\left(D_{a_{0} \ldots a_{i}}\right) .
$$

Then, Proposition 2.4 of [Lei08] and our Proposition 2.15 imply the result. If $\mathcal{D}$ is a filter cover of $C$, then $\mathcal{D}^{\text {op }}$ is an ideal cover of $C^{\text {op }}$. Since the equality $\chi(X)=\chi\left(X^{\text {op }}\right)$ holds for any finite category $X$ having Euler characteristic, we obtain the desired formula.

Note that the order on the index set is not essential, since we can choose a total order on any finite set, and Proposition 2.9 also holds in the case of reduced Čech nerves.

Corollary 3.4. Let both $A$ and $B$ be filters or ideals of a finite category $C$. If each of $A, B, A \cap B$, and $A \cup B$ has Euler characteristic, then we have

$$
\chi(A \cup B)=\chi(A)+\chi(B)-\chi(A \cap B) .
$$




\section{References}

[DI04] Daniel Dugger and Daniel C. Isaksen. Topological hypercovers and $\mathbb{A}^{1}$ realizations. Math. Z. 246 (2004), no. 4, 667-689.

[FLS11] Thomas M. Fiore, Wolfgang Luck, and Roman Sauer. Euler characteristics of categories and homotopy colimits. Doc. Math. 16 (2011), 301-354.

[Hir03] Philip S. Hirschhorn. Model Categories and Their Localizations. Math. Surveys Monogr., Vol. 99. American Mathematical Society, Providence, RI, 2003, xvi+457 pp.

[Lei08] Tom Leinster. The Euler characteristic of a category. Doc. Math. 13 (2008), 21-49.

[Qui73] Daniel Quillen. Higher algebraic K-theory. In: Algebraic K-Theory I: Higher K-Theories (Proc. Conf., Battelle Memorial Inst., Seattle, Wash., 1972), Lecture Notes in Math., Vol. 341. Springer, Berlin, 1973, pp. 85147.

[Rot64] Gian-Carlo Rota. On the foundations of combinatorial theory. I. Theory of Möbius functions. Z. Wahrsch. Verw. Gebiete 2 (1964), 340-368.

[Seg68] Graeme Segal. Classifying spaces and spectral sequences. Publ. Math. Inst. Hautes Ètudes Sci., 34 (1968), 105-112.

[Sta12] Richard P. Stanley. Enumerative Combinatorics. Volume 1. Second edition. Cambridge Stud. Adv. Math., Vol. 49. Cambridge University Press, Cambridge, 2012, xiv+626 pp.

[Tho79] R.W. Thomason. Homotopy colimits in the category of small categories. Math. Proc. Cambridge Philos. Soc. 85 (1979), no. 1, 91-109.

[Zap98] Roman R. Zapatrin. Algebraic duality for partially ordered sets. Pure Math. Appl. 9 (1998), nos. 3-4, 485-490.

Kohei Tanaka tanaka@shinshu-u.ac.jp

Institute of Social Sciences, School of Humanities and Social Sciences, Academic Assembly, Shinshu University, 3-1-1, Matsumoto, Nagano, 390-8621, Japan 